\title{
The Medical Device Unique Device Identifier as the Single Source of Truth in Healthcare Enterprises - Roadmap for Implementation of the Clinically Integrated Supply Chain
}

\author{
James E Tcheng (1D' \\ Miriam V Nguyen' \\ Helen W Brann' \\ Patricia A Clarke (D) \\ Maureen Pfeiffer' \\ Jane R Pleasants ${ }^{2}$ \\ Gregory W Shelton' \\ Joseph F Kelly (D) \\ 'Department of Medicine, Duke \\ University Hospital and Health System, \\ Durham, NC, USA; ${ }^{2}$ Strategic \\ Marketplace Initiative, Westborough, \\ MA, USA
}

\begin{abstract}
Documentation and tracking of supplies, equipment and medical devices is central to operational, financial, and clinical aspects of safe, efficient, and effective patient care. The labeling of medical devices with a unique device identifier (UDI) creates the opportunity to tightly integrate device information across health information systems by using the UDI as the index "source of truth". Across 3 hospitals of the Duke University Health System, we executed a comprehensive implementation of UDI-based device and supply information management in our cardiac catheterization and electrophysiology laboratories. Following are our key insights. Implementing a UDI-centric environment is a complex undertaking requiring integration of information systems, management processes, and clinical workflows involving leadership, inventory management, supply chain, clinical and billing teams. Implementation involves the domains of procedure documentation, electronic health records (EHRs), charge capture and billing, and interface and information technology systems, including information systems vendors. Replacing manual processes with electronic messages is not simply an exercise in programming information systems - successful execution requires orchestrated re-engineering of clinical and operational workflows. Our initiative resulted in a more efficient and effective supply chain, eliminated operational and clinical documentation errors, automated the posting of device implant data to the EHR, reduced clinician burden, improved charge capture, and produced a substantial financial benefit, with return on investment recognized in well under 1 year. We believe our stepwise approach to accomplishing a clinically integrated supply chain can serve as a roadmap for other healthcare enterprises to follow.
\end{abstract}

Keywords: unique device identifier, medical devices, supply chain, clinical documentation, implementation roadmap, interoperability

\section{Introduction}

The United States Food and Drug Administration (FDA) Unique Device Identification System for medical devices was created with multiple public health objectives, including reducing medical errors, simplifying device data management in health information systems, facilitating device identification in the context of adverse events, returning performance information to manufacturers, expediting recalls, improving FDA device-related safety communications, enabling patients and consumers, and benefitting healthcare enterprise operations. ${ }^{1}$ The FDA Unique
Correspondence: James E Tcheng Duke University Hospital and Health System, Hock Plaza Suite 403, 2424 Erwin Road, Durham, NC, 27705, USA

Tel $+1919668-8796$

Email tchen00I@mc.duke.edu 
Device Identifier (UDI) Final Rule was published in 2013, resulting in availability of a human-readable, machineinterpretable UDI (typically a barcode) on all moderate and high-risk (Class II and Class III) and implantable devices by $2017 .^{2}$ However, 8 years after the FDA UDI Final Rule, use of the UDI in clinical workflows at the point of care is still substantially lacking; in particular, clinical documentation (eg, procedure reports) remains largely verbose without notation or use of the UDI. While the potential clinical, research, and financial advantages have been clearly described, ${ }^{3-5}$ actualization of the UDI-enabled healthcare system is distant.

For the opportunities of UDI to be realized, capture of the UDI at point of use is a logical prerequisite; labelling and packaging of supplies and devices is by itself insufficient. In addition to capturing the UDI at the point of use, the even larger challenge is to coordinate and replace the multiple redundant processes and data sources for managing supply and device information across inventory management, supply chain, operational documentation, clinical documentation, and charge capture processes and systems with a coordinated, reliable environment using the UDI as the single "source of truth". To be successful, the environment should also reduce clinician and staff burden. A predicate UDI demonstration project, Building UDI into Longitudinal Device Evaluation (BUILD), identified full integration across operational, clinical, and financial domains as the key framework. ${ }^{6,7}$ To address the challenge, in 2017 our health system leadership identified UDI implementation as a high priority project. Using a "people, processes, and technology" rubric, ${ }^{8}$ we successfully converted to a UDI-centric environment for managing device and supply information in our cardiac catheterization and electrophysiology laboratories. The purpose of this report is to describe the approach we followed, discuss the timelines, requisite resources, and lessons learned, and articulate our roadmap as a potential model for other healthcare enterprises to follow.

\section{Organizational Context}

The Duke University Health System is comprised of the Duke University School of Medicine, Duke University School of Nursing, the Duke Clinic, and member hospitals. The flagship hospital, Duke University Hospital, is a full-service tertiary and quaternary care hospital located in Durham, North Carolina. Duke University Hospital has 957 licensed inpatient beds and admitted over 42,000 patients in fiscal year 2020. The Duke University Hospital Cardiac Catheterization and Electrophysiology Laboratories deliver a full portfolio of diagnostic and therapeutic cardiovascular procedures. Two additional Health System hospitals, Duke Regional Hospital and Duke Raleigh Hospital, also provide cardiac catheterization and electrophysiology services.

Foundational to the UDI initiative, Duke University Health System had in place a robust supply chain management system (SAP, SAP America, Inc., Newtown Square, PA), including a highly curated item master list and mature supply ordering processes. Both the cardiovascular procedure reporting system (ApolloLX, Lumedx, Oakland, CA) and electronic health record (Epic, Epic Systems, Verona, WI) were mutually used across the Health System and had been operational for a decade. Message handling was the province of an integration broker (HealthShare Health Connect, InterSystems, Cambridge, Ma). Prior to UDI implementation, inventory was managed with a combination of manual processes and a standalone inventory management system.

\section{People}

Four champions led the initiative. The overall UDI champion was an interventional cardiologist-informatician and Director, Duke Information Systems for Cardiovascular Care who was passionate about the clinical benefits and operational value of the UDI. He was an investigator of the BUILD demonstration project. ${ }^{6,7}$ Additional champions included the Health System Vice President for Supply Chain, Health System Vice President for the Heart Center, and Health System Administrative Director for Cardiac Catheterization and Electrophysiology Laboratories. A complete list of key personnel and responsibilities can be found in Table 1.

\section{Problems and Goals}

The overarching goal was to create a coordinated environment of accurate, consistent, and reproducible supply and medical device information linking clinical care, supply chain, and billing processes. The alternative, maintaining the status quo, meant continuing with manual, error-prone, duplicative workflows, including gaps in communication and information sharing between clinicians and supply chain teams. Particularly relevant were the processes for reconciliation of inventory, procedure documentation, and EHR systems when items were inaccurately entered or failed to match across information systems, resulting in billing delays 
Table I Key UDI Initiative Personnel

\begin{tabular}{|l|l|}
\hline Position & Responsibilities \\
\hline UDI Champion & Overall project leader \\
Vice President - Heart Center & Service line administrative champion responsible for funding, resource coordination \\
Vice President - Supply Chain & Assigned resources to integrate supply chain information systems and inventory management processes \\
Laboratories Administrative Director & Logistics and workflow transformation \\
Heart Center Administrative Director & Overall coordination of project \\
Project Manager & Project management, initiative tracking \\
Business Analyst & Workflow documentation, interface messaging specifications, business rules \\
Technical Lead & Interface architecture, assurance of high availability \\
Inventory Manager & Supplies processing, inventory workflow \\
Supply Chain Manager & Integration of supply chain and inventory management systems \\
Integration Broker (team) & Messaging services for UDI data exchange \\
Super Users (team) & Evaluation of proposed workflows, user acceptance testing, rollout support \\
Vendors & Vendor-specific messaging and specifications (SAP, CareFusion, Epic, Lumedx, HealthShare) \\
\hline
\end{tabular}

and reimbursement rejections. Moreover, each hospital had its own separate teams and processes for validating supplies and devices usage for charge capture. The overall project goal was to replace our former non-UDI environment with barcode scanning coupled with the electronic communication of supply and device usage based on the UDI to all relevant systems and processes. Furthermore, the goal was to be inclusive of all disposables, supplies, and devices - not just the documentation of implanted medical devices. Specifically, the project targeted the following.

- Replace manual inventory management processes with UDI-based automated inventory management (receipt, stocking, usage, replenishment)

- Automate capture of supply and device usage data via barcode scanning at the point of use

- Electronically communicate accurate, high-quality supply and device usage "source of truth" data to the procedure documentation system (for listing of supplies and disposables in procedure logs and physician procedure reports), to the EHR system (for insertion into the device implant table), to the charge capture and billing system, and to the supply chain management system (for re-ordering and par level management)

- Reduce physician and staff documentation and workflow burden

- Optimize the financial margin related to supplies and devices

To accomplish the transformation, four distinct phases of work were required: 1) strategy setting - the executive process resulting in formal approval of the project; 2) planning - identifying and defining effort, resources, and specifications; 3) technical build - configuring and testing information technology components and corresponding changes to workflows; and 4) deployment - knowledge dissemination and conversion to the new environment.

\section{Process - Strategy Setting}

In the first stage of the initiative, we established high-level goals, articulating the framework, defining the scope, and outlining the project plan among stakeholders. This stage was orchestrated by the overall UDI (physician) champion in collaboration with the Heart Center Administrative Director over a period of approximately 2 months. The cardiac catheterization and electrophysiology laboratories were identified as the locus of the initiative for the following reasons: a high volume of cases, supplies and devices already having a UDI printed on packaging, fewer logistical barriers than the operating room - particularly a lack of barriers to barcode scanning at point of use - and an agile clinical procedure documentation system that could be readily configured to utilize the UDI.

This stage included directed discussions among leadership of the laboratories, supply chain, health system and Heart Center information technology groups, and external vendors (SAP, Lumedx, and Epic). High-level agreement about initiative responsibilities were determined and documented. Technical aspects were identified, with development of detailed specifications assigned as future projects in the respective work queues. A project manager was selected to organize and accomplish the initiative. 


\section{Process - Planning}

The second stage was an iterative process to specify details and determine costs of the project. This stage produced detailed project plans featuring explicit workflow, dataflow, and interface specification documents and required approximately 3 months to complete. The bulk of the project was estimated to require 500 person-hours of effort distributed across 15-20 individuals. This culminated in review and approval by the Heart Center Information Technology, Health System New Technology, and Health System Financial and Capital Planning governance committees.

- A project outline identified goals and requirements, stakeholders, executive sponsors, operational and technical teams, and expected high-level outcomes.

- Meetings were held with leadership and staff of the cardiac catheterization and electrophysiology laboratories and representatives of supply chain management, inventory management, and charge capture teams to understand current workflows and anticipate the future state. Each group articulated their desired vision, specifically identifying end-user interactions with the projected UDI-aligned systems and processes.

- An information flow diagram was developed (Figure 1) via meetings with stakeholders (supply chain, hospital information systems, cardiology informatics, inventory management, coding and billing, interface, and information systems vendors). Critical was identification of data sources (eg, SAP item master index for device information, Epic Radiant module for procedure scheduling and patient demographic data, inventory management system for UDI data) and data recipients (eg, Lumedx for structured clinical documentation, ${ }^{9}$ SAP for inventory replenishment). The resulting documentation included technical requirements, constraints, and dependencies, including delineation of roles and responsibilities of each of the parties.

- Our existing inventory management system was evaluated to determine suitability for meeting the design requirements. The conclusion of that evaluation dictated

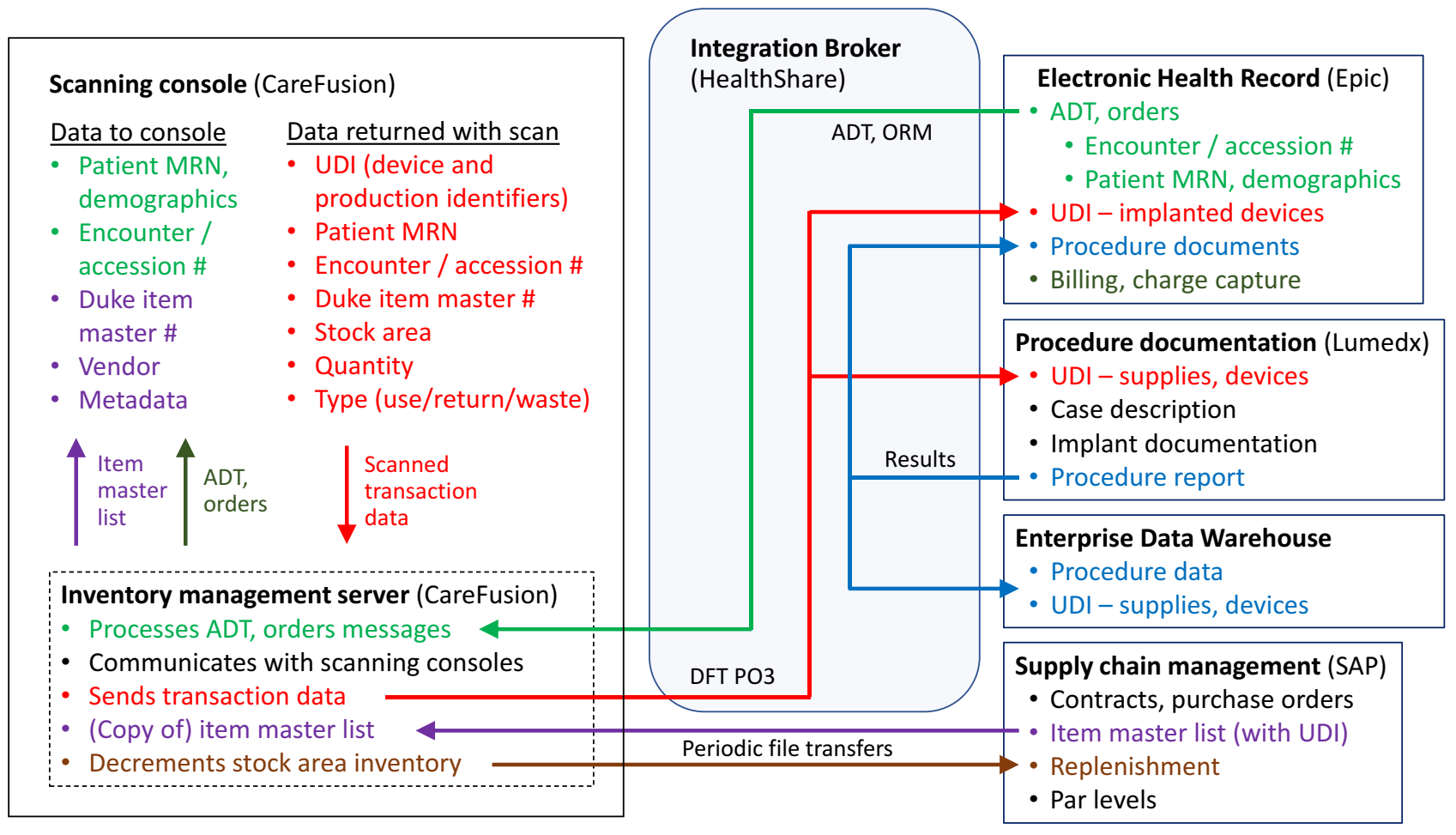

Figure I Use of the Unique Device Identifier in the Cardiac Catheterization and Electrophysiology Laboratories. The diagram depicts the dataflows among the 5 primary information technology systems. Clinically relevant dataflows are from the electronic health record to the inventory management system to initialize the patient (green text and lines) and flow of scanned UDI data and associated metadata from the inventory management system to the procedure documentation system, electronic health record, and enterprise data warehouse (red text and lines). Supply chain and inventory management system handling of device and supply information is represented with the purple and brown text and lines at the bottom of the Figure.

Abbreviations: UDI, Unique Device Identifier; ADT, Admission, Discharge, and Transfer; ORM, HL7 Order Message; DFT PO3, HL7 Detail Financial Transaction - Post Order 3. 
that an alternative system would be needed. A competitive analysis identified CareFusion Pyxis (Becton Dickinson and Company, Franklin Lakes, NJ) as the system best meeting our specifications.

- Several barcode scanning systems were evaluated by stakeholders from the perspectives of space, usability, use of barcode data standards, and readiness for data exchange. The ability of the barcode scanning system to read and convert the UDI format regardless of UDI issuing agency (GS1, Health industry Business Communications Council, or International Council for Commonality in Blood Banking Automation) was confirmed. Of note, the UDIs of supplies and devices utilized in the cardiac catheterization and electrophysiology laboratories are issued by GS1.

- Formal project documents were finalized (ie, technical specifications, effort requirements, timelines, costs), reviewed, and approved by oversight committees.

\section{Technology - Technical Build}

Based on the dataflows (Figure 1), this third stage featured the technical build required for each of the 6 core information systems to share and utilize UDI and associated data. The technical build accounted for 3 months of the project and included extensive end-to-end testing to assure data fidelity and consistent processing across systems, including expected production workflows as well as out-of-norm scenarios. Integrated and functional testing for the project required approximately 30 hours. Batch controls in the EHR proved critical to address the timeliness of $\log$ posts and facilitate timely edits of supplies on an asneeded basis. Following is additional detail about selected aspects of the technical build.

- Deployment of a new inventory management system. During the planning process, we determined that our existing standalone inventory management system would not meet the data exchange requirements central to a UDI-enabled environment. We elected to install an alternative inventory management system (CareFusion Pyxis) that was already operational in other areas of the hospital, adding the catheterization and electrophysiology laboratories as nodes to the existing installation. The installation still required new inventory workstations, barcode scanners, and dedicated (virtualized) test and production servers, along with additional system redundancy to assure high availability.

- Changes to user interfaces. User-facing screens in the inventory management system (CareFusion) and the procedure documentation system (Lumedx) were modified to reflect capture and use of the UDI (eg, display of the UDI, expired inventory alerts, key device-associated metadata, clinically meaningful device name).

- "Source of truth" assignments. Detailed meetings with information system representatives specified the full complement of source data necessary to enable a UDI-centric environment.

- Epic - source of transaction data, particularly patient demographics and procedure scheduling information, along with data requirements for billing and for populating the device implant table

- SAP - source of supply chain data, including the item master list, par levels, device pricing, and specifications for inventory replenishment

- CareFusion - source of inventory receipt, use, and procedure-level (point of care) inventory management data

- Lumedx - clinical consumer of the UDI, with device metadata (eg, manufacturer, clinically meaningful device name, clinically relevant size) being automatically inserted into procedure report documentation

- New interfaces. Although robust messaging existed throughout our enterprise, new Health Level 7 (HL7) v2 interfaces were required to support the dataflows. Message exchange was accomplished using an integration broker (HealthShare).

- HL7 DFT PO3 transaction messages - device vended and used, returned, or wasted messages from CareFusion to Epic and from CareFusion to Lumedx

○ Inventory use data, item master list - automated file transfers were established from CareFusion to SAP (conveying usage information for reordering) and from SAP to CareFusion (reflecting updates to the item master list)

- HL7 ORM order messages - ORM general order messages to convey order encounter/accession numbers for procedure transactions to associate and route order information to the appropriate Epic and Lumedx procedure level encounter 
- HL7 ADT messages - admission, discharge, and transfer orders from Epic to CareFusion to provide a patient list and store hospital encounter information, and to facilitate manual case setup during downtime

\section{Deployment}

For deployment, we chose to transition wholesale to the new environment at Duke University Hospital on a specific date and time. For several weeks before cutover, personnel were educated and trained regarding changes to their respective workflows. The weekend before cut-over, the inventory team performed a "yearend" inventory, updated the inventory master list in the inventory management system, set up new stock locations, and scanned equipment transactions into the supply stations. Laboratory staff were trained on-site using a demonstration unit and tip sheets, with super users and vendors providing hands-on support on the day of golive. A command center was initiated a few days before go-live and maintained for several days following deployment. Separately, Duke Regional Hospital and Duke Raleigh Hospital transitioned to the UDI-centric approach at a deployment cadence of 2 and 4 months, respectively, following the above framework.

\section{Metrics and Results}

Based upon the implementation science framework of feasibility, penetration, fidelity, acceptability, adoption, sustainability, and cost, ${ }^{10}$ results of our transformation include the following.

- The project was executed as projected and within budget, with a capital cost of less than $\$ 20,000$ and approximately 500 person-hours of effort

- Cut-over to UDI-based workflows and dataflows occurred without technical or operational glitches and has been embraced by all personnel without incident

- The UDI-centric environment has reduced clinical documentation errors and supply chain management issues related to supply and device data issues to negligible levels

- In the electrophysiology laboratory, replacement of manual transcription of device information, parameters, and settings for cardiac implanted electronic devices (eg, pacemakers, implantable cardioverter- defibrillators) with electronically interfaced data indexed by the UDI is saving 15-20 minutes of staff documentation time per device implant procedure

- Device implant information is being automatically inserted in the EHR device implant table, facilitating clinical communication along with access by patients to their device implant information

- The updated workflows for reviewing and posting charges have streamlined and standardized these processes across all hospitals of the Health System (Table 2), with inventory management, procedure documentation, and EHR information systems supply and device data now being completely consistent, resulting in elimination of rejected charges based on inconsistent or incorrect supply or device information

- In terms of return on investment, tighter management of par levels coupled with consistent charge capture of all supplies and medical devices has resulted in an increase in revenue recognition of over $\$ 600,000$ per year (comparison of technical revenues received in FY 2019 for the cardiac catheterization and electrophysiology laboratories compared with the average of FY 2016 and FY 2017)

- Device and supply data are now available in the enterprise data warehouse for quality assessment, process improvement, clinical research, and other query-based uses (eg, the UDI has already been used to manage recall of a pacemaker lead)

\section{Discussion - and Lessons for the Field}

This was a complex initiative that required coordination and alignment across multiple internal and external groups. Principal hurdles were addressed as follows.

\section{Knowledge Gap}

Resistance to change was encountered during discussions with leadership and stakeholders, typically expressed by the queries "why?" and "what's in it for me?". For many, the potential of the UDI was made poignant by reflecting on the ubiquitous marking of consumer products with the Universal Product Code and in particular the grocery store checkout process. Clinical and inventory management staff were concerned about the operational changes and intimated that they would quickly return to old processes if the new workflows increased 
Table 2 Impact of Unique Device Identifier on Charge Capture Processes

\begin{tabular}{|c|c|c|}
\hline $\begin{array}{l}\text { Impact on Charge } \\
\text { Capture }\end{array}$ & Relevant Best Practices & Specific Approaches Applied \\
\hline $\begin{array}{l}\text { Use of Data Originating } \\
\text { from External } \\
\text { Information Systems }\end{array}$ & $\begin{array}{l}\text { - Utilize logic and edit checks to identify potential data } \\
\text { errors } \\
\text { - Post charges automatically as soon as data payload is } \\
\text { complete }\end{array}$ & $\begin{array}{l}\text { - UDI as the "source of truth" for supplies and devices, } \\
\text { shared with inventory management, supply chain, electro- } \\
\text { nic health record, and procedure documentation systems } \\
\text { - Integration of UDI into charge capture work queue as a } \\
\text { dependency for coding and billing }\end{array}$ \\
\hline Employee Education & $\begin{array}{l}\text { - Provide educational materials and training to revenue } \\
\text { personnel regarding information systems, applicable } \\
\text { guidelines, and regulations } \\
\text { - Establish centralized policies regarding charge capture }\end{array}$ & $\begin{array}{l}\text { - Durable educational materials } \\
\text { - Liaison representing staff perspectives } \\
\text { - Education and training using a learning management system } \\
\text { - Hands-on training } \\
\text { - Help desk }\end{array}$ \\
\hline $\begin{array}{l}\text { Process } \\
\text { Documentation }\end{array}$ & $\begin{array}{l}\text { - Develop workflows and troubleshooting protocols } \\
\text { that are straightforward to follow by staff and } \\
\text { management } \\
\text { - Create process bridges to ensure connections } \\
\text { between clinical and financial domains and enable } \\
\text { the clinically integrated supply chain } \\
\text { - Maintain communication channels between clinical } \\
\text { and financial teams }\end{array}$ & $\begin{array}{l}\text { - Frequent meetings for staff and leadership to articulate } \\
\text { issues and envision future state } \\
\text { - Internal policy reviews } \\
\text { - Quarterly information dissemination }\end{array}$ \\
\hline $\begin{array}{l}\text { Reconciliation and } \\
\text { Quality Assurance } \\
\text { Auditing }\end{array}$ & $\begin{array}{l}\text { - Conduct reviews of professional and technical } \\
\text { charges } \\
\text { - Assess clinical documentation for completeness and } \\
\text { compliance } \\
\text { - Review causes of delays in charge posting compared } \\
\text { with industry benchmarks }\end{array}$ & $\begin{array}{l}\text { - Daily reconciliation of professional, technical, and supply } \\
\text { charges for all cases } \\
\text { - Monthly quality assurance audits of a percentage of cases } \\
\text { - Yearly audits by hospital finance } \\
\text { - Monthly root cause analysis of late and denied charges } \\
\text { - Incorporation of process improvements }\end{array}$ \\
\hline
\end{tabular}

Abbreviation: UDI, Unique Device Identifier.

burden or proved unreliable. Issues related to inertia were addressed by using approaches developed in the domain of implementation science, ${ }^{11-13}$ including open communication, intense stakeholder engagement, user-centric testing, and the provision of on-site training and hands-on support during golive. Repetitive, consistent communication of the benefits of a UDI-enabled environment proved critical to the success of the initiative.

\section{Resourcing}

The initiative required Heart Center, enterprise, and vendor resources. Funding for hardware and software was negotiated through Heart Center capital processes. Formal project management facilitated the early delineation of roles and responsibilities of the affected information technology groups, resulting in coordinated allocation of resources and assignment of responsibilities that accomplished the individual components of the initiative.

\section{Conversion to a "Source of Truth"}

While conceptually straightforward, the use of the UDI and associated metadata across the 6 separate information systems required extensive coordination. Fundamental was delineation of precisely how the UDI could be maximally leveraged by each system to replace manually managed supply and device information and facilitate process automation. For example, while a robust item master list is maintained in our supply chain system (SAP), device product names in that system reflect manufacturer branding rather than clinical nomenclature. For supply chain and inventory management processes, we continued to use the product name as specified by the manufacturer, linking the product name with the corresponding UDI. But for clinical purposes, a clinical product name (again linked to the UDI) was substituted as the source text for embedding the device name into the procedure log and physician procedure reports. 


\section{Information Systems Limitations}

The capabilities and limitations of each information system had to be discovered and managed. In the ideal setting, bidirectional messaging would effortlessly occur among all systems of the UDI, associated metadata, and device status (eg, implanted, wasted, or unable to deploy). For example, no process existed in our EHR for removing an implant from the device table if an error occurred during scanning. The EHR also did not provide administrative control to correct linked orders, which resulted in separate events in downstream systems and mismatches in scanned supplies and clinical events. Processes for mitigating and correcting these limitations had to be created and implemented.

\section{Identifying the UDI on Package Labeling}

With most device packages having multiple barcodes, identifying the UDI barcode proved frustrating to clinical staff. This has required ongoing staff training and remains a limitation that can delay the finalization of clinical documentation and billing. Feedback regarding this issue has been provided to the FDA, including a specific request for labeling improvements to clearly identify the UDI on package labeling. While graphical symbol marking to identify the UDI has been approved as a standard by the International Standards Organization (ISO $15223 ; 1: 2021),{ }^{14}$ this standard is voluntary and subject to adoption by device and supply manufacturers. Until mandatory marking is required (likely by regulation), the easy identification of the UDI on packaging will likely remain a limitation.

\section{Conclusions}

We believe that a UDI-enabled environment can be implemented by any health system. UDI implementation requires coordination across multiple disciplines and the engagement of champions representing each of those disciplines. Extensive and detailed project planning will help assure success, including the coordination of goals, timelines, and resources through formal project management. The tight integration of workflow and dataflow is key for UDI to be embraced by staff as an improvement over the historical approach. The UDI implementation should be all-inclusive, specifically linking together inventory management, supply chain, clinical, and financial domains. We trust that the roadmap described herein can be used by healthcare enterprises to catalyze success.

\section{Disclosure}

All authors have no conflicts of interest in this work.

\section{References}

1. U.S. Food and Drug Administration. Unique device identification system. A rule by the food and drug administration on 9/24/2013. Federal register 2013-23059; 2013. Available from: https://www.fed eralregister.gov/documents/2013/09/24/2013-23059/unique-deviceidentification-system. Accessed March 1, 2021.

2. U.S. Food and Drug Administration. Unique device identification system (UDI System); 2020. Available from: https://www.fda.gov/ medical-devices/device-advice-comprehensive-regulatory-assistance /unique-device-identification-system-udi-system. Accessed March 1, 2021.

3. Shuren J, Califf RM. Need for a national evaluation system for health technology. JAMA. 2016;316(11):1153-1154. doi:10.1001/ jama.2016.8708

4. Sherman RE, Anderson SA, Dal Pan GJ, et al. Real-world evidence what is it and what can it tell us? $N$ Engl J Med. 2016;375 (23):2293-2297. doi:10.1056/NEJMsb1609216

5. Statement from FDA Commissioner Scott Gottlieb, M.D., on new efforts to enhance and modernize the FDA's approach to medical device safety and innovation. U.S. Food \& Drug Administration. 2018. Available from: https://www.fda.gov/NewsEvents/Newsroom/ PressAnnouncements/ucm604672.htm. Accessed March 1, 2021.

6. Tcheng JE, Crowley J, Tomes M, et al.; MDEpiNet UDI Demonstration Expert Workgroup. Unique device identifiers for coronary stent postmarket surveillance and research: a report from the Food and Drug Administration Medical Device Epidemiology Network Unique Device Identifier demonstration. Am Heart J. 2014;168(4):405-413.e2. doi:10.1016/j.ahj.2014.07.001

7. Wilson N. Building UDI into Longitudinal Data for Medical Device Evaluation (BUILD). Point of care capture of UDI for implantable devices. Final summary report \& roadmap; 2019. Available from: http://mdepinet.org/wp-content/uploads/BUILD-Update.pdf. Accessed March 1, 2021.

8. Morgan JM, Liker JK. The Toyota Product Development System. Integrating People, Process, and Technology. Boca Raton, FL.: Productivity Press; 2006.

9. Sanborn TA, Tcheng JE, Anderson HV, et al. ACC/AHA/SCAI 2014 health policy statement on structured reporting for the cardiac catheterization laboratory: a report of the American College of Cardiology Clinical Quality Committee. J Am Coll Cardiol. 2014;63 (23):2591-2623. doi:10.1016/j.jacc.2014.03.020

10. Proctor E, Silmere H, Raghavan R, et al. Outcomes for implementation research: conceptual distinctions, measurement challenges, and research agenda. Adm Policy Ment Health. 2011;38(2):65-76. doi:10.1007/s10488-010-0319-7

11. Neuman HB, Kaji AH, Haut ER. Practical guide to implementation science. JAMA Surg. 2020;155(5):434-435. doi:10.1001/ jamasurg.2019.5149

12. Bauer MS, Damschroder L, Hagedorn H, Smith J, Kilbourne AM. An introduction to implementation science for the non-specialist. $B M C$ Psychol. 2015;3(1):32. doi:10.1186/s40359-015-0089-9

13. Handley MA, Gorukanti A, Cattamanchi A. Strategies for implementing implementation science: a methodological overview. Emerg Med J. 2016;33(9):660-664. doi:10.1136/emermed-2015-205461

14. ISO. ISO 15223-1:2021. Medical devices - symbols to be used with information to be supplied by the manufacturer. Available from: https://iso.org/standard/77326.html. Accessed November 25, 2021. 


\section{Publish your work in this journal}

Medical Devices: Evidence and Research is an international, peerreviewed, open access journal that focuses on the evidence, technology, research, and expert opinion supporting the use and application of medical devices in the diagnosis, monitoring, treatment and management of clinical conditions and physiological processes. The identification of novel devices and optimal use of existing devices which will lead to improved clinical outcomes and more effective patient management and safety is a key feature of the journal. The manuscript management system is completely online and includes a very quick and fair peer-review system. Visit http:// www.dovepress.com/testimonials.php to read real quotes from published authors. 